\title{
Does Clopidogrel have an Effect on Migraine Attacks that Emerge after Closure of Atrial Septal Defects?
}

\author{
Klopidogrelin Atrial Septal Defekt Kapatılması Sonrası Ortaya Çıkan Migren Atakları \\ Üzerine Etkisi var m1?
}

\author{
Halil İbrahim Akçay, Murat Kürtüncü \\ İstanbul University Faculty of Medicine, Department of Neurology, İstanbul, Turkey
}

\section{Does Clopidogrel have an Effect on Migraine Attacks that Emerge after Closure of Atrial Septal Defects?}

New-onset migraine attacks occur in $15 \%$ of patients after transcatheter atrial septal closure. In these patients, migraine attacks start during the initial days or weeks. Antithrombotic treatment with aspirin is used for 6 months after closure. Early observational cross-sectional studies demonstrated that addition of clopidogrel to aspirin was associated with less frequent and milder migraine attacks.

The Clopidogrel for the Prevention of New-onset Migraine Headache Following Transchateter Closure of Atrial Septal Defects study by Rodés-Cabau et al. (1) was performed in 6 Canadian centers and included patients with atrial septal defect (ASD) aged 18 years and above who had a clinical indication for closure. Patients who had previous migraine, allergy or intolerance to antithrombotic drugs who needed anticoagulation, were pregnant or breastfeeding, or had a previous unsuccessful ASD closure procedure were excluded. Some patients only took aspirin $80 \mathrm{mg} /$ day and the others took aspirin $80 \mathrm{mg} /$ day and clopidogrel $75 \mathrm{mg} /$ day. This treatment protocol was initiated one day before the ASD closure procedure and continued for 3 months. The patients did not receive a clopidogrel loading dose at the beginning. The patients kept a headache diary. The Migraine Disability Assessment Questionnaire was used to assess severity of headache. All migraine questionnaires and headache diaries were evaluated by two neurologists. The primary end point in this study was determined as the number of new-onset migraine attacks within 3 months of the ASD closure procedure. Secondary endpoints were the incidence of new-onset migraine attacks, the number of migraine attacks in the $1^{\text {st }}$ and $3^{\text {rd }}$ months, severity of migraine attacks, and time to the first migraine attack. The study included 291 patients who were treated between
2008-2014; 71 patients were excluded because they had a previous history of migraine. A total of 171 patients formed the study groups. Among them, 87 patients took aspirin plus placebo (the placebo group) and 84 patients took aspirin plus clopidogrel (the clopidogrel group). The results of the study showed that the mean monthly migraine number was lower in the clopidogrel group.

In summary, dual antiplatelet treatment for the initial three months after transcatheter ASD closure significantly decreased the number of new-onset migraine attacks and the probability of their occurrence. Moderate to severe migraine attacks were not seen in patients who were taking the dual treatment; however, one third of patients who only took aspirin had severe and disabling migraine attacks. Future research is needed to determine whether a longer duration of treatment is necessary. In another study, Spencer et al. (2) showed significant benefits of clopidogrel that was given before transcatheter closure on migraine in patients with patent foramen ovale who also had migraine. The benefits of specific antithrombotic treatments such as clopidogrel on decreasing occurrence of migraine attacks supports the idea that platelet and coagulation cascades are important in migraine pathogenesis. Other studies also showed that anticoagulant and antiplatelet (clopidogrel) use in special patient groups decreased migraine attacks. However, two randomized studies found no benefits of warfarin and clopidogrel in decreasing the frequency of migraine attacks $(3,4)$.

This study showed that clopidogrel is highly protective against migraine attacks in the presence of specific triggers. Increased platelet aggregation was shown after ASD closure and as a result, release of pro-inflammatory cytokines such as interleukin (IL)-1, IL-6, and IL-8 and substances such as serotonin increased. These substances were found to be associated with migraine attacks. Clopidogrel has an antiplatelet effect in addition to antioxidant and anti-inflammatory features. The vaso-protective effect of 
clopidogrel by adenosine phosphate receptor blockage may play a role in preventing migraine attacks.

\section{Ethics}

Peer-review: Internal peer-reviewed.

\section{References}

1. Rodés-Cabau J, Horlick E, Ibrahim R, Cheema AN, Labinaz M, Nadeem N, Osten M, Côté M, Marsal JR, Rivest D, Marrero A, Houde C. Effect of clopidogrel and aspirin vs aspirin alone on migraine headaches after transcatheter atrial septal defect closure: The CANOA randomized clinical trial. JAMA 2015;314:2147-2154.

2. Spencer BT, Qureshi Y, Sommer RJ. A retrospective review of clopidogrel as primary therapy for migraineurs with right to left shunt lesions. Cephalalgia 2014;34:933-937.

3. Wammes-van der Heijden EA, Smidt MH, Tijssen CC, van't Hoff AR, Lenderink AW, Egberts AC. Effect of low-intensity acenocoumarol on frequency and severity of migraine attacks. Headache 2005;45:137-143.

4. Chambers JB, Seed PT, Ridsdale L. Clopidogrel as prophylactic treatment for migraine: a pilot randomised, controlled study. Cephalalgia 2014;34:1163-1168. 